\title{
Resveratrol attenuates azidothymidine-induced cardiotoxicity by decreasing mitochondrial reactive oxygen species generation in human cardiomyocytes
}

\author{
RACHEL YUE GAO ${ }^{1 *}$, PARTHA MUKHOPADHYAY ${ }^{1 *}$, RAJESH MOHANRAJ $^{1}$, HUA WANG $^{2}$, \\ BÉLA HORVÁTH ${ }^{1,3}$, SHI YIN ${ }^{2}$ and PÁL PACHER ${ }^{1}$
}

\begin{abstract}
${ }^{1}$ Section on Oxidative Stress Tissue Injury, Laboratory of Physiological Studies, and ${ }^{2}$ Laboratory of Liver Diseases, National Institute on Alcohol Abuse and Alcoholism, National Institutes of Health, Bethesda, MD 20892, USA;

${ }^{3}$ Institute of Human Physiology and Clinical Experimental Research, Semmelweis University, Budapest, Hungary
\end{abstract}

Received July 8, 2010; Accepted October 18, 2010

DOI: $10.3892 / \mathrm{mmr} .2010 .390$

\begin{abstract}
Nucleotide reverse transcriptase inhibitors, such as zidovudine (azidothymidine, AZT) and stavudine, represent a class of approved antiretroviral agents for highly active antiretroviral therapy, which prolongs the life expectancy of patients infected with human-immunodeficiency virus. Unfortunately, the use of these drugs is associated with known toxicities in the liver, skeletal muscle, heart and other organs, which may involve increased reactive oxygen species (ROS) generation, among other mechanisms. Resveratrol is a polyphenolic plant-derived antioxidant abundantly found in certain grapes, roots, berries, peanuts and red wine. This study, using primary human cardiomyocytes, evaluated the effects of AZT and pre-treatment with resveratrol on mitochondrial ROS generation and the cell death pathways. AZT induced concentration-dependent cell death, involving both caspase-3 and -7 and poly(ADP-ribose) polymerase activation, coupled with increased mitochondrial ROS generation in human cardiomyocytes. These effects of AZT on mitochondrial ROS generation and cell death may be attenuated by resveratrol pre-treatment. The results demonstrate that mitochondrial ROS generation plays a pivotal role in the cardiotoxicity of AZT in human cardiomyocytes, and resveratrol may provide a potential strategy to attenuate these pathological alterations, which are associated with widely used antiretroviral therapy.
\end{abstract}

Correspondence to: Dr Pál Pacher, Section on Oxidative Stress Tissue Injury, Laboratory of Physiological Studies, National Institutes of Health/NIAAA, 5625 Fishers Lane, MSC-9413, Room 2N-17, Rockville, MD 20852, USA

E-mail: pacher@mail.nih.gov

*Contributed equally

Key words: resveratrol, azidothymidine, cell death, cardiomyocytes, reactive oxygen species

\section{Introduction}

Nucleotide reverse transcriptase inhibitors, such as zidovudine (azidothymidine, AZT) and stavudine, represent a class of approved antiretroviral agents for highly active antiretroviral therapy, which prolongs the life expectancy of patients infected with human-immunodeficiency virus (HIV). Unfortunately, the use of these drugs is associated with known toxicities in various organs, including the liver, skeletal muscle and heart. HIV-positive patients are at risk of developing end-organ damage, including cardiomyopathy, which is seemingly becoming increasingly prevalent (1). Cardiomyopathy manifests as dilated cardiomyopathy, or as isolated left or right ventricular dysfunction; both are associated with a poor prognosis, resulting in symptomatic heart failure in up to $5 \%$ of HIV patients (1). The precise mechanism of HIV-associated cardiomyopathy has yet to be elucidated. It may involve direct myocardial infection with HIV, post-infective cardiac autoimmunity, nutritional deficiencies, or the cardiotoxic effects of antiretroviral drugs, such as AZT (1). Indeed, numerous preclinical reports implicate the direct cardiotoxic effects of AZT in various rodent models. This may involve multiple mechanisms, including the generation of reactive oxygen species (ROS) in cardiomyocytes and endothelial cells (2-12).

Resveratrol (3,4',5-trihydroxystilbene), a plant-derived polyphenolic compound belonging to a class of stilbenes (abundantly found in certain grapes, roots, berries and peanuts) was recently shown to exert various cardiovascular protective effects in pre-clinical models of myocardial ischemic reperfusion injury and atherosclerosis $(13,14)$, metabolic diseases (15-17), and in aged mice (18-21). In this study, we explored the effects of AZT on mitochondrial ROS generation and associated cell death (both apoptotic and necrotic) using human primary cardiomyocytes, and evaluated the effects of resveratrol on these pathological processes.

\section{Materials and methods}

Reagents and cell culture. AZT and resveratrol were purchased from Sigma Chemicals (St. Louis, MO, USA). 

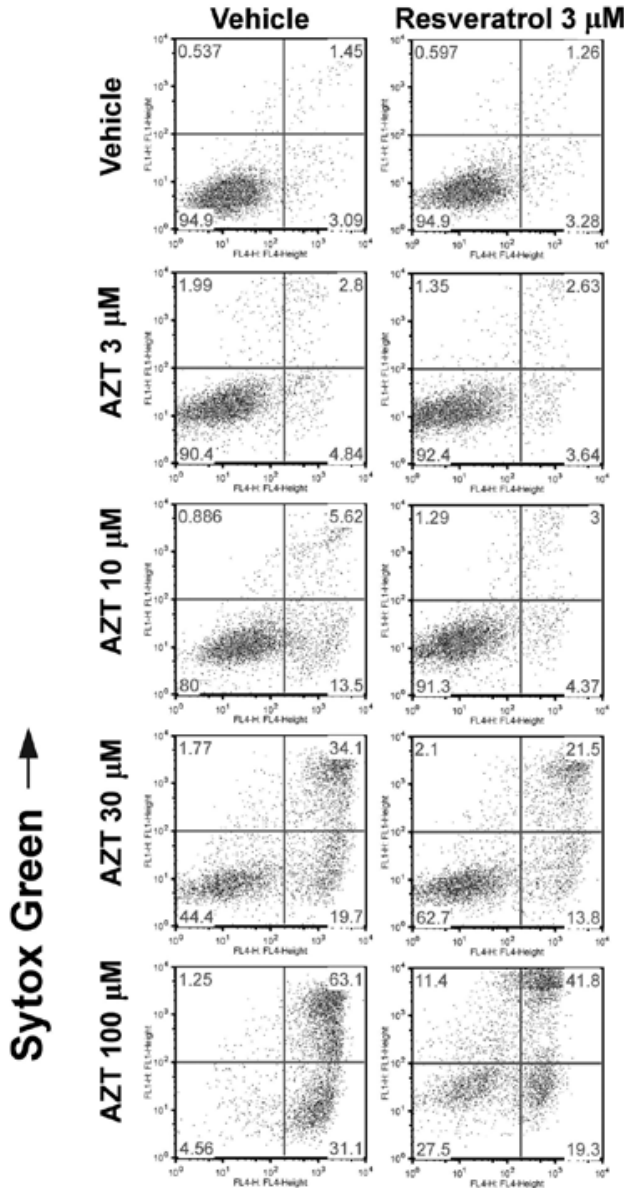

Annexin V-APC $\rightarrow$
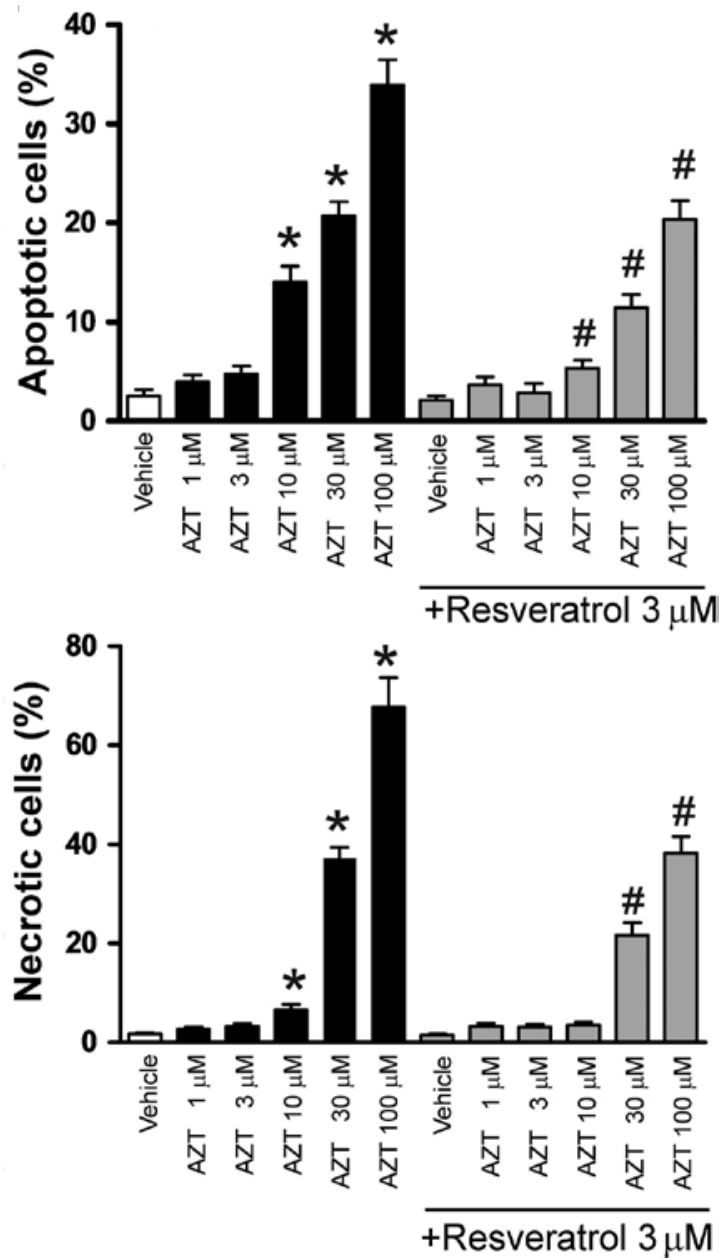

Figure 1. Resveratrol attenuates AZT-induced cell death in human cardiomyocytes. Human cardiomyocytes were pre-treated with or without various concentrations of resveratrol for $2 \mathrm{~h}$, followed by treatment with various doses of AZT with or without resveratrol. After $48 \mathrm{~h}$, the cells were harvested for flow cytometric analysis with Annexin V for apoptosis and Sytox Green for necrosis. Left panel, representative flow cytometry charts demonstrating the effects of AZT with or without resveratrol pre-treatment on cell death (apoptotic and necrotic) in human primary cardiomyocytes; right panel, the quantification of apoptotic and necrotic cell death from the left panel. Values represent the means $\pm \mathrm{SEM}(\mathrm{n}=4)$. ${ }^{*} \mathrm{P}<0.05$, vehicle vs. AZT; ${ }^{\#} \mathrm{P}<0.05$, AZT with or without resveratrol, respectively.

Human cardiomyocytes (HCM) were obtained from ScienCell Research Laboratories (Carlsbad, CA, USA) and cultured in polylysine-coated plates using complete cardiac myocyte medium according to the manufacturer's recommendation as previously described (22). HCM were treated with AZT as indicated in cardiomyocyte growth medium for $48 \mathrm{~h}$.

Simultaneous determination of cell death and mitochondrial superoxide/ROS generation. Mitochondrial superoxide/ROS generation and cell death were determined as previously described $(23,24)$. Briefly, the cells were loaded with MitoSOX Red (Invitrogen, Carlsbad, CA, USA) for $30 \mathrm{~min}$, followed by trypsinization as well as staining with APC-Annexin-V and Sytox Green (Invitrogen). The samples were run on a flow cytometer with $488 \mathrm{~nm}$ excitation to measure oxidized MitoSOX Red in the FL2 and FL3 channels, APC-Annexin V (FL4) and Sytox Green (FL1). Data were also collected from the FSC (forward scatter), SSC (side scatter) and FL1 and FL4 channels. The cells were plotted for FSC and SSC. Cell debris with low FSC and SSC were excluded from the analysis. The cells were then analyzed for APC-Annexin V (FL4) and Sytox
Green (FL1). Cells that exhibited apoptosis (FL-4-positive) or were dead (FL-1-positive) were excluded from the analysis, and MitoSOX Red staining was analyzed for each population as a histogram of mean intensity (FL2). Thus, MitoSOX Red of the cells analyzed excluded any non-specific interferences from apoptotic and dead cells (23).

Caspase-3 and -7 activity. The activity of caspase-3 and -7 was measured using the Apo-one Homogeneous Caspase-3/7 assay kit (Promega), according to the manufacturer's instructions (25).

Poly(ADP-ribose) polymerase activity. Poly(ADP-ribose) polymerase (PARP) activity was assayed by colorimetry according to the manufacturer's instructions, as previously described (Trevigen) (25).

Statistical analysis. Results are reported as the means \pm SEM. P-values $<0.05$ were considered significant. Statistical analysis between two measurements were determined using the twotailed unpaired Student's t-test. 

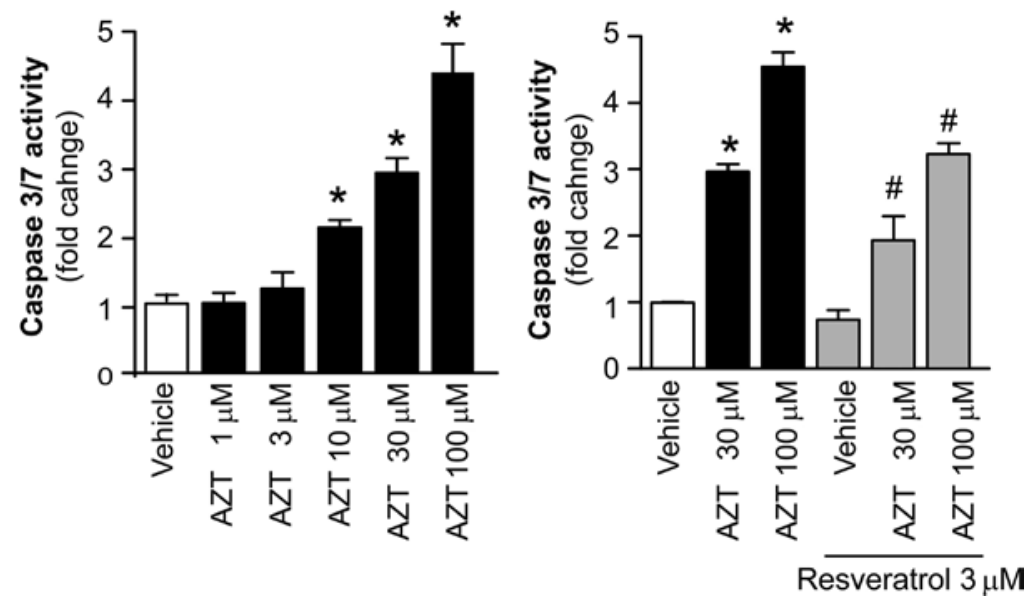

Figure 2. Resveratrol attenuates the AZT induction of caspase-3 and -7 activity in human cardiomyocytes. Human cardiomyocytes were treated with resveratrol and AZT, as described in Fig. 1. The cells were harvested and caspase-3 and -7 activity was measured. Left panel, the concentration-dependent effect of AZT on caspase-3 and -7 activity in human cardiomyocytes; right panel, the effects of AZT with or without resveratrol pre-treatment. Values represent the means \pm SEM (n=3-6). "P<0.05, vehicle vs. AZT; ${ }^{\sharp} \mathrm{P}<0.05$, AZT with or without resveratrol, respectively.
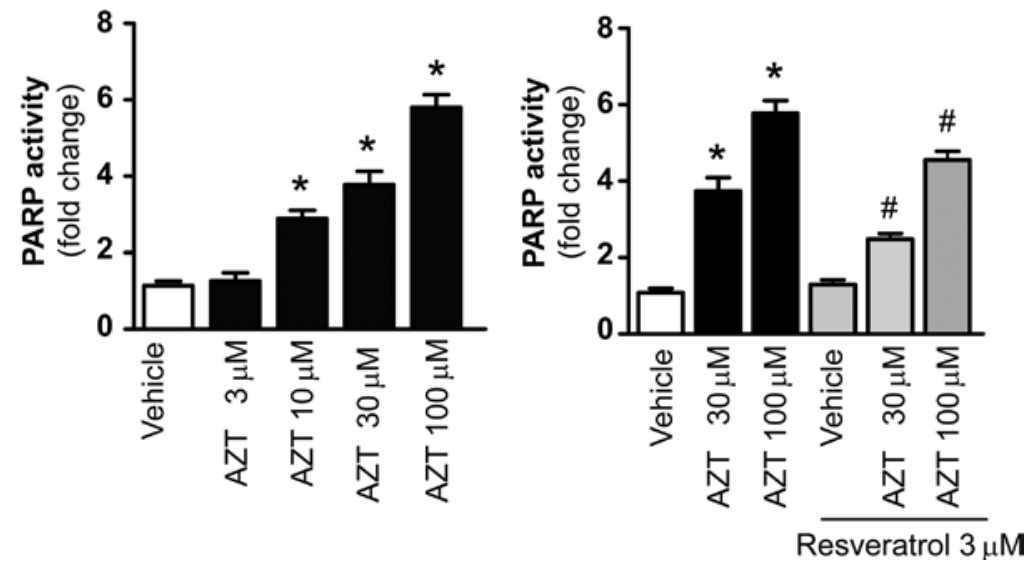

Figure 3. Resveratrol attenuates the AZT induction of PARP activity in human cardiomyocytes. Human cardiomyocytes were treated as described in Fig. 1. The cells were harvested and PARP activity was measured. Left panel, the concentration-dependent effect of AZT on PARP activity in human cardiomyocytes; right panel, the effects of AZT with or without resveratrol pre-treatment. Values represent the means $\pm \mathrm{SEM}(\mathrm{n}=8)$. ${ }^{*} \mathrm{P}<0.05$, vehicle vs. AZT; ${ }^{*} \mathrm{P}<0.05$, AZT with or without resveratrol, respectively.

\section{Results}

Resveratrol attenuates azidothymidine-induced cell death in human cardiomyocytes. Primary cardiomyocytes were treated with various concentrations of AZT for $48 \mathrm{~h}$. Cell apoptosis and necrosis were then determined by flow cytometric analysis. AZT treatment increased cell apoptosis and necrosis in human primary cardiomyocytes in a concentration-dependent manner. Pre-treatment with $3 \mu \mathrm{M}$ resveratrol attenuated the AZT-induced cell apoptosis and necrosis (Fig. 1).

Resveratrol attenuates azidothymidine-induced caspase- 3 and -7 activity in human cardiomyocytes. To further understand the underlying mechanisms of AZT-induced cardiomyocyte apoptosis and the prevention of such cell death by resveratrol, caspase-3 and -7 activity, which is indicative of cell apoptosis was measured. AZT treatment induced the elevation of caspase- 3 and -7 activity in human primary cardiomyocytes in a concentration-dependent manner. Pre-treatment with resveratrol prevented AZT-induced caspase-3 and -7 activity (Fig. 2).
Resveratrol attenuates azidothymidine-induced PARP activity in human cardiomyocytes. The activation of PARP has been shown to play an important role in inducing necrotic cell death. We investigated whether AZT-induced cell death is mediated via the activation of PARP. AZT treatment increased PARP activity in human primary cardiomyocytes in a concentrationdependent manner. Resveratrol pre-treatment significantly attenuated the AZT-induced increase in PARP activity (Fig. 3).

Resveratrol attenuates azidothymidine-induced mitochondrial reactive oxygen species/superoxide generation in human cardiomyocytes. Mitochondria are one of the major cellular producers of ROS, and increased ROS generation promotes the activation of various cell death pathways. Thus, AZT-induced cell death in human cardiomyocytes was examined to determine whether it involves increased mitochondrial ROS generation. AZT treatment significantly increased mitochondrial ROS generation in human primary cardiomyocytes in a concentration-dependent manner, which was attenuated by resveratrol pre-treatment (Fig. 4). 


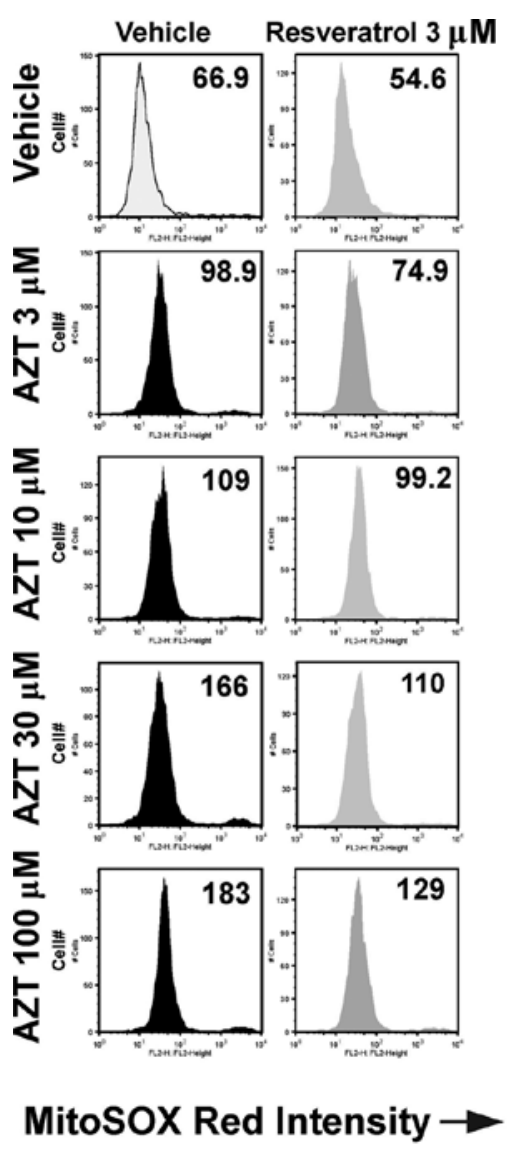

\section{Discussion}

In this study, using primary human cardiomyocytes, we demonstrated that mitochondrial ROS generation and the consequent activation of various cell death pathways contribute to the cardiotoxicity of the retroviral agent AZT. We also demonstrated that resveratrol pre-treatment was capable of attenuating AZT-induced mitochondrial ROS generation and the activation of interrelated cell death pathways.

The cardiotoxic effects of antiretroviral drugs such as AZT have been implicated in the development of HIV-associated cardiomyopathy in humans (1). Indeed, AZT induces cardiomyopathy in various rodent model systems. The cardiotoxicity of AZT is complex and likely involves multiple interrelated mechanisms, such as the inhibition of cardiac mitochondrial DNA polymerase- $\gamma$ (4), alterations in pyrimidine deoxyribonucleotide pools by the inhibition of thymidine kinase 2 (7), increased myocardial oxidative stress and attenuated antioxidant defense $(5,6,9,26,27)$, oxidative damage in cardiac mitochondria $(2,3)$, increased endothelial cell oxidative stress and mitochondrial dysfunction $(8,11)$, and activation of the nuclear enzyme PARP (9). Supporting the role of oxidative stress in the development of AZT-induced cardiomyopathy, transgenic mitochondrial superoxide dismutase and mitochondrially-targeted catalase prevent antiretroviral drug-induced oxidative stress and cardiomyopathy in mice (12).

Consistent with these reports, we demonstrated increased concentration-dependent mitochondrial ROS generation by AZT in human cardiomyocytes. Increased ROS generation in cardiomyocytes may trigger the activation of various mito-

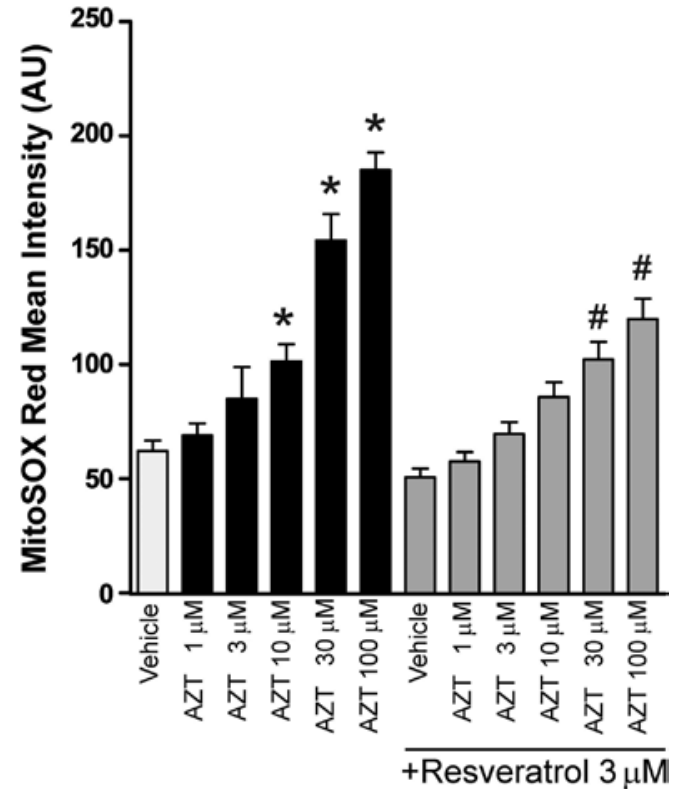

Figure 4. Resveratrol attenuates AZT-induced mitochondrial ROS generation in human cardiomyocytes. Human cardiomyocytes were treated as described in Fig. 1. The cells were harvested and analyzed by flow cytometric analysis using MitoSOX Red dye. Left panel, representative flow cytometry charts demonstrating the effects of AZT with or without resveratrol pre-treatment on mitochondrial ROS generation in human primary cardiomyocytes; right panel, the quantification of MitoSox Red mean intensity from the left panel ( 3 experiments). Values represent the means \pm SEM $(n=4) .{ }^{*} \mathrm{P}<0.05$, vehicle vs. AZT; ${ }^{\text {P }}<0.05$, AZT with or without resveratrol, respectively.

chondrial-dependent and -independent cell death pathways involved in apoptotic and necrotic cell death (e.g., activation of caspases and PARP-1) (28). Furthermore, superoxide in the mitochondria may react with nitric oxide to generate a highly reactive oxidant, peroxynitrite (29), which may impair cellular function and lead to cell death $(25,30)$ and/or dysfunction (31) in cardiomyocytes via multiple interrelated mechanisms. Indeed, AZT induced caspase-3 and -7 and PARP-dependent cell death in cardiomyocytes.

Numerous recent studies provide evidence that resveratrol treatment attenuates myocardial ischemic reperfusion injury and atherosclerosis (13), and improves endothelial function in rodent models of type 2 diabetes $(21,32,33)$, as well as in aged mice and rats $(21,34)$. The multiple synergistic effects of resveratrol are likely to be responsible for its cardiovascular protective potential, which may include up-regulation of eNOS, increased NO bioavailability $(32,35,36)$, promotion of mitochondrial biogenesis (37), down-regulation of TNF- $\alpha$ and inhibition of NADPH oxidases (32), inhibition of NF- $\kappa \mathrm{B}$ (38-40) and attenuation of mitochondrial ROS generation (33), among others. Consistent with its antioxidant properties, resveratrol treatment attenuated the AZT-induced mitochondrial ROS generation and the activation of caspase-3 and -7 and the PARP-dependent cell death pathways in human cardiomyocytes.

Collectively, these results demonstrate that AZT induces increased mitochondrial ROS generation in human cardiomyocytes, triggering the activation of caspase- 3 and -7 and the PARP-1-dependent cell death pathways. AZT-induced cell death is attenuated by resveratrol pre-treatment, which 
decreases mitochondrial ROS generation, providing a potential strategy for the attenuation of these antiretroviral-therapy associated pathological alterations.

\section{Acknowledgements}

This study was supported by the Intramural Research Program of the National Institutes of Health/NIAAA (to P.P.). B.H. is a recipient of a Fellowship from the Hungarian Scientific Research Foundation: NKTH-OTKA-EU (MB08A-80238).

\section{References}

1. Currie PF and Boon NA: Immunopathogenesis of HIV-related heart muscle disease: current perspectives. AIDS 17 (Suppl 1): 21-28, 2003.

2. De la Asuncion JG, Del Olmo ML, Gomez-Cambronero LG, Sastre J, Pallardo FV and Vina J: AZT induces oxidative damage to cardiac mitochondria: protective effect of vitamins $\mathrm{C}$ and $\mathrm{E}$. Life Sci 76: 47-56, 2004.

3. Lewis W, Papoian T, Gonzalez B, et al: Mitochondrial ultrastructural and molecular changes induced by zidovudine in rat hearts. Lab Invest 65: 228-236, 1991.

4. Lewis W, Simpson JF and Meyer RR: Cardiac mitochondrial DNA polymerase-gamma is inhibited competitively and noncompetitively by phosphorylated zidovudine. Circ Res 74: 344-348, 1994.

5. Mak IT, Goldfarb MG, Weglicki WB and Haudenschild CC: Cardiac pathologic effects of azidothymidine (AZT) in Mg-deficient mice. Cardiovasc Toxicol 4: 169-177, 2004.

6. Mak IT, Nedelec LF and Weglicki WB: Pro-oxidant properties and cytotoxicity of AZT-monophosphate and AZT. Cardiovasc Toxicol 4: 109-115, 2004

7. Morris GW, Iams TA, Slepchenko KG and McKee EE: Origin of pyrimidine deoxyribonucleotide pools in perfused rat heart: implications for 3'-azido-3'-deoxythymidine-dependent cardiotoxicity. Biochem J 422: 513-520, 2009.

8. Sutliff RL, Dikalov S, Weiss D, et al: Nucleoside reverse transcriptase inhibitors impair endothelium-dependent relaxation by increasing superoxide. Am J Physiol Heart Circ Physiol 283: $\mathrm{H} 2363-\mathrm{H} 2370,2002$.

9. Szabados E, Fischer GM, Toth K, et al: Role of reactive oxygen species and poly-ADP-ribose polymerase in the development of AZT-induced cardiomyopathy in rat. Free Radic Biol Med 26: 309-317, 1999.

10. Freyssenet D, DiCarlo M, Escobar P, Grey J, Schneider J and Hood DA: Zidovudine (AZT) induced alterations in mitochondrial biogenesis in rat striated muscles. Can J Physiol Pharmacol 77: 29-35, 1999.

11. Kline ER, Bassit L, Hernandez-Santiago BI, et al: Long-term exposure to AZT, but not d4T, increases endothelial cell oxidative stress and mitochondrial dysfunction. Cardiovasc Toxicol 9: 1-12, 2009

12. Kohler JJ, Cucoranu I, Fields E, et al: Transgenic mitochondrial superoxide dismutase and mitochondrially targeted catalase prevent antiretroviral-induced oxidative stress and cardiomyopathy. Lab Invest 89: 782-790, 2009.

13. Das DK and Maulik N: Resveratrol in cardioprotection: a therapeutic promise of alternative medicine. Mol Interv 6: 36-47, 2006

14. Baur JA and Sinclair DA: Therapeutic potential of resveratrol: the in vivo evidence. Nature Reviews 5: 493-506, 2006.

15. Sharma S, Anjaneyulu M, Kulkarni SK and Chopra K: Resveratrol, a polyphenolic phytoalexin, attenuates diabetic nephropathy in rats. Pharmacology 76: 69-75, 2006.

16. Thirunavukkarasu M, Penumathsa SV, Koneru S, et al: Resveratrol alleviates cardiac dysfunction in streptozotocininduced diabetes: role of nitric oxide, thioredoxin, and heme oxygenase. Free Radic Biol Med 43: 720-729, 2007.

17. Zang M, Xu S, Maitland-Toolan KA, et al: Polyphenols stimulate AMP-activated protein kinase, lower lipids, and inhibit accelerated atherosclerosis in diabetic LDL receptor-deficient mice. Diabetes 55: 2180-2191, 2006.

18. Barger JL, Kayo T, Vann JM, et al: A low dose of dietary resveratrol partially mimics caloric restriction and retards aging parameters in mice. PLoS ONE 3: e2264, 2008.
19. Baur JA, Pearson KJ, Price NL, et al: Resveratrol improves health and survival of mice on a high-calorie diet. Nature 444: 337-342, 2006.

20. Csiszar A, Labinskyy N, Jimenez R, et al: Anti-oxidative and anti-inflammatory vasoprotective effects of caloric restriction in aging: role of circulating factors and SIRT1. Mech Ageing Dev 130: 518-527, 2009.

21. Pearson KJ, Baur JA, Lewis KN, et al: Resveratrol delays age-related deterioration and mimics transcriptional aspects of dietary restriction without extending life span. Cell Metab 8: 157-168, 2008.

22. Mukhopadhyay P, Rajesh M, Batkai S, et al: CB1 cannabinoid receptors promote oxidative stress and cell death in murine models of doxorubicin-induced cardiomyopathy and in human cardiomyocytes. Cardiovasc Res 85: 773-784, 2010.

23. Mukhopadhyay P, Rajesh M, Hasko G, Hawkins BJ, Madesh M and Pacher P: Simultaneous detection of apoptosis and mitochondrial superoxide production in live cells by flow cytometry and confocal microscopy. Nat Protoc 2: 2295-2301, 2007.

24. Mukhopadhyay P, Rajesh M, Yoshihiro K, Hasko G and Pacher P: Simple quantitative detection of mitochondrial superoxide production in live cells. Biochem Biophys Res Commun 358: 203-208, 2007.

25. Mukhopadhyay P, Rajesh M, Batkai S, et al: Role of superoxide, nitric oxide, and peroxynitrite in doxorubicin-induced cell death in vivo and in vitro. Am J Physiol Heart Circ Physiol 296: H1466-H1483, 2009.

26. Weglicki WB, Chmielinska JJ, Tejero-Taldo I, et al: Neutral endopeptidase inhibition enhances substance $\mathrm{P}$ mediated inflammation due to hypomagnesemia. Magnes Res 22: S167-S173, 2009.

27. Papparella I, Ceolotto G, Berto L, et al: Vitamin C prevents zidovudine-induced $\mathrm{NAD}(\mathrm{P}) \mathrm{H}$ oxidase activation and hypertension in the rat. Cardiovasc Res 73: 432-438, 2007.

28. Pacher P and Szabo C: Role of poly(ADP-ribose) polymerase 1 (PARP-1) in cardiovascular diseases: the therapeutic potential of PARP inhibitors. Cardiovasc Drug Rev 25: 235-260, 2007.

29. Pacher P, Beckman JS and Liaudet L: Nitric oxide and peroxynitrite in health and disease. Physiol Rev 87: 315-424, 2007.

30. Levrand S, Vannay-Bouchiche C, Pesse B, et al: Peroxynitrite is a major trigger of cardiomyocyte apoptosis in vitro and in vivo. Free Radic Biol Med 41: 886-895, 2006.

31. Pacher P, Schulz R, Liaudet L and Szabo C: Nitrosative stress and pharmacological modulation of heart failure. Trends Pharmacol Sci 26: 302-310, 2005.

32. Zhang H, Zhang J, Ungvari Z and Zhang C: Resveratrol improves endothelial function: role of TNF alpha\} and vascular oxidative stress. Arterioscler Thromb Vasc Biol 29: 1164-1171, 2009.

33. Ungvari Z, Labinskyy N, Mukhopadhyay P, et al: Resveratrol attenuates mitochondrial oxidative stress in coronary arterial endothelial cells. Am J Physiol Heart Circ Physiol 297: H1876-H1881, 2009.

34. Ungvari Z, Orosz Z, Rivera A, et al: Resveratrol increases vascular oxidative stress resistance. Am J Physiol 292: H2417-H2424, 2007.

35. Bradamante S, Barenghi L, Piccinini F, et al: Resveratrol provides late-phase cardioprotection by means of a nitric oxideand adenosine-mediated mechanism. Eur J Pharmacol 465: $115-123,2003$

36. Taubert D and Berkels R: Upregulation and activation of eNOS by resveratrol. Circulation 107: e78-79, 2003.

37. Csiszar A, Labinskyy N, Pinto JT, et al: Resveratrol induces mitochondrial biogenesis in endothelial cells. Am J Physiol Heart Circ Physiol 297: H13-H20, 2009.

38. Yeung F, Hoberg JE, Ramsey CS, et al: Modulation of NF-kappaB-dependent transcription and cell survival by the SIRT1 deacetylase. Embo J 23: 2369-2380, 2004

39. Csiszar A, Smith K, Labinskyy N, Orosz Z, Rivera A and Ungvari Z: Resveratrol attenuates TNF-\{alpha\}-induced activation of coronary arterial endothelial cells: role of NF- $\{k a p p a\} B$ inhibition. Am J Physiol 291: H1694-H1699, 2006.

40. Csiszar A, Labinskyy N, Podlutsky A, et al: Vasoprotective effects of resveratrol and SIRT1: attenuation of cigarette smoke-induced oxidative stress and proinflammatory phenotypic alterations. Am J Physiol Heart Circ Physiol 294: H2721-H2735, 2008. 